\title{
Auditing stillbirths at Lower Umfolozi War Memorial Regional Hospital: A 12-month review
}

\author{
I Govender, MB ChB, MMed, FCPHM, Dip Obst (SA) \\ KwaZulu-Natal Department of Health, Lower Umfolozi War Memorial Regional Hospital, Empangeni, South Africa
}

Corresponding author: I Govender (indira.govender@gmail.com)

\begin{abstract}
Background. Although the total number of stillbirths worldwide was estimated at 2.6 million in 2009, there is currently a dearth of literature on stillbirths in developing countries and rural settings, where the majority of such births occur. The 'Hands Up' Mortality and Morbidity Extraction Tool (HUMMET), developed at Lower Umfolozi War Memorial Regional Hospital (LUWMRH) in 2010, outlines a systematic approach to summarising individual cases of adverse perinatal outcomes.

Objectives. To depict the HUMMET form by describing the detailed demographic and obstetric profile of patients who delivered a stillborn infant at LUWMRH, as well as risk factors associated with these stillbirths between 1 April 2014 and 31 March 2015. The findings add to a global initiative advanced by the Lancet series on stillbirths, aimed at raising awareness of stillbirth statistics in low- and middle-income countries.

Methods. A total of 310 detailed stillbirth case summaries of 305 patients were collected during the study period, representing $90 \%$ of the total number of stillborn infants delivered at LUWMRH. A retrospective audit of the HUMMET forms was conducted and the cases were further summarised in a Microsoft Excel spreadsheet that allowed for a univariate analysis of the variables.

Results. The stillbirth rate at LUWMRH is much higher than that at other regional hospitals owing to the number of at-risk referrals and emergency cases from surrounding clinics and district hospitals. Referrals were from local clinics (49\%) and district hospitals (45\%), 35\% of stillbirths were due to abruptio placentae and a large proportion were associated with gestational hypertension, pre-eclampsia and/or eclampsia. Avoidable factors were predominantly a late patient response to reduced fetal movements and delays in transfer to hospital. Twenty percent of stillbirths were associated with inappropriate monitoring or management of the obstetric condition at the district hospital. Conclusion. The HUMMET form provides a systematic approach to analysing cases of perinatal morbidity and mortality in line with the requirements of the Perinatal Problem Identification Programme database, but provides more details on the circumstances and contributing factors. A repeat audit is recommended to determine whether interventions have been effective.
\end{abstract}

S Afr Med J 2017;107(12):1121-1126. DOI:10.7196/SAMJ.2017.v107i12.12491

The World Health Organization (WHO) defines a stillbirth as a baby born with no signs of life at or after 28 weeks' gestation, or with a birth weight of at least 1000 g. ${ }^{[1]}$ In 2009 , the total number of stillbirths worldwide was estimated at 2.6 million, but there is not much literature on stillbirths from developed countries and even less from developing countries. ${ }^{[2,3]}$ Most stillbirths (98\%) occur in low- and middle-income countries - two-thirds in rural families. ${ }^{[4]}$ However, global policy appears to reflect social stigma and beliefs, as these figures go unmentioned in the United Nations Millennium Development Goals, the Global Burden of Disease study or routine WHO reports. ${ }^{[5]}$ In addition to risk factors associated with stillbirths in developed countries (i.e. prior stillbirth, minority status, low socioeconomic status, maternal thinness, older maternal age, single marital status, and smoking, alcohol and drug use), maternal infections, such as syphilis and malaria, and prolonged labour resulting in asphyxia, trauma and infection, have been associated with stillbirths in these countries. ${ }^{[6]}$ Circumstances known to be more common in rural areas and developing countries, such as socioeconomic deprivation, inadequate antenatal care and lack of education, have also been shown to increase the risk of stillbirth. ${ }^{[7,8]}$

In South Africa (SA), the Perinatal Problem Identification Programme (PPIP) includes a stillborn baby weighing $\geq 500 \mathrm{~g}$ as part of the total stillbirth rate (SBR). The Saving Babies 2012 - 2013 report, based on data collected for the PPIP, reported SA's SBR and rate for stillborn infants weighing $\geq 1000 \mathrm{~g}$ (SBR $\geq 1000 \mathrm{~g}$ ) as 23.1 and 17.6 per 1000 total births, respectively. ${ }^{[9]}$ The SBR and SBR $\geq 1000 \mathrm{~g}$ for
KwaZulu-Natal (KZN) are 26.2 and 19.7 per 1000 total deliveries, and those for regional hospitals in the 2012 - 2013 Saving Babies report 30.2 and 22.6, respectively.

Lower Umfolozi War Memorial Regional Hospital (LUWMRH), located in the semi-rural part of northern KZN, specialises in maternal and child health services and covers a population of 2.3 million in three health districts that are among the poorest in the country: uThungulu, Umkhanyakude and Zululand. ${ }^{[10]}$ Even though LUWMRH is a regional hospital, it provides a unique combination service, receiving direct referrals from 21 local clinics, and functions as the local district hospital. It provides a tertiary service for 16 district hospitals in the three districts that comprise Region 4 of KZN. Using the average rates for the three districts, the $\mathrm{SBR}$ and $\mathrm{SBR} \geq 1000 \mathrm{~g}$ for Region 4 are 23.4 and 18.0 per 1000 total deliveries, respectively.

Daily perinatal audit meetings are conducted during the week and all stillbirths and adverse perinatal outcomes are reviewed in detail. Individual maternity case records are summarised using a paper-based form known as the 'Hands Up' Mortality and Morbidity Extraction Tool (HUMMET) (Appendix A), which was developed by the hospital's head of department for obstetrics and gynaecology in 2010 and implemented at all facilities in the region's three referring health districts.

Auditing the HUMMET forms began as an internal qualityimprovement project in the obstetrics and gynaecology department to provide more detailed information on stillbirths compared with those in the PPIP database, and subsequently to inform targeted 
interventions aimed at reducing stillbirths in the service area. The audit also served to highlight the practical use of HUMMET for summarising cases of perinatal morbidity and mortality. It adds to a global initiative advanced by the Lancet series on stillbirths aimed at raising awareness of stillbirth statistics in low- and middle-income countries. $^{[5]}$

\section{Methods}

Records of the hospital's PPIP database from 1 April 2014 to 31 March 2015 show that there were 8795 deliveries, including 346 stillborn infants, of whom 249 weighed $>1000 \mathrm{~g}$ and 97 weighed between $500 \mathrm{~g}$ and $999 \mathrm{~g}$. There were 310 corresponding HUMMET forms for this period, representing $90 \%$ of the total number of stillbirths reported in the PPIP database.

The HUMMET form is used to summarise all cases of maternal deaths, stillbirths, early and late neonatal deaths, cases of birth asphyxia and infants with a 5-minute Apgar score of $<7$. The lefthand side of the form is divided into sections that describe the basic demographic details and obstetric history of the antenatal patient, including her antenatal clinic visits, problems during antenatal care, labour, delivery and the postpartum period, as well as birth weight and delivery time. On the right-hand side of the form healthcare workers can analyse the case and identify risk factors, avoidable factors, substandard care/missed opportunities, learning opportunities and actions to be taken with regard to the patient and the health service. The main factors contributing to each perinatal death are coded according to the PPIP and entered into the electronic PPIP database.

A retrospective audit was conducted of all 310 HUMMET forms collected during the 12-month review period. The information on the forms was further summarised on a Microsoft Excel spreadsheet into categories corresponding to basic demographic details of patients at the time of delivery, antenatal history and risk factors, descriptions of the stillbirths and mode of delivery, avoidable factors surrounding the stillbirth, and identifiable areas of substandard care relating to the management of the pregnancy. Thereafter a univariate analysis of the categorical and ordinal variables was performed on the summarised data to produce descriptive statistics. The stillbirths at LUWMRH were then disaggregated to their referring districts and used to recalculate new SBRs for the respective districts, using the SBR data captured on the District Health Information System (DHIS) for the study period.

\section{Ethical approval}

Ethical approval to conduct the audit was granted by LUWMRH's ethics committee and the Umgungundlovu Health District Review Board (ref. no. UHERB160303).

\section{Results}

Table 1 shows a comparison of the SBR and SBR $\geq 1000 \mathrm{~g}$ data during the study period at LUWMRH with those in the 9th Saving Babies report. ${ }^{[9]}$

Table 2 shows the number of stillbirths at LUWMRH per referring district hospital in Region 4 during the study period. The cases referred from Ekombe and Nkandla hospitals were combined, as these are neighbouring hospitals. As Mbongolwane Hospital is known to refer certain emergency cases to Eshowe Hospital, these two facilities were also combined.

Table 3 compares the SBR in a facility per district during the study period, as captured on the DHIS with an adjusted SBR, which includes referred stillbirth cases from Umkhanyakude and Zululand districts during the same period.

\begin{tabular}{lcl}
\multicolumn{2}{l}{ Table 1. Comparison of stillbirth rates } \\
\hline Location & SBR & SBR $\geq \mathbf{1 ~ 0 0 0 ~} \mathbf{~}$ \\
\hline South Africa & 23.1 & 17.6 \\
KwaZulu-Natal & 26.2 & 19.7 \\
Regional hospitals & 30.2 & 22.6 \\
LUWMRH & 39.3 & 28.3 \\
Region 4 & 23.4 & 18.0
\end{tabular}

SBR = stillbirth rate; LUWMRH $=$ Lower Umfolozi War Memorial Regional Hospital.

Table 2. Stillbirths per district hospital in Region $4^{\star}, n$

\begin{tabular}{ll}
\hline District and hospital & Stillbirths, $\boldsymbol{n}$ \\
\hline Umkhanyakude district & \\
Bethesda Hospital & 8 \\
Hlabisa Hospital & 23 \\
Manguzi Hospital & 3 \\
Mosvold Hospital & 10 \\
Mseleni Hospital & 4 \\
Total & 48 \\
Uthungulu district & \\
Catherine Booth Hospital & 7 \\
Ekombe and Nkandla hospitals & 8 \\
Eshowe and Mbongolwane hospitals & 14 \\
Kwamagwaza Hospital & 8 \\
22 clinics & 151 \\
Total & 188 \\
Zululand district & \\
Benedictine Hospital & 11 \\
Ceza Hospital & 3 \\
Itshelejuba Hospital & 9 \\
Nkonjeni Hospital & 14 \\
Vryheid Hospital & 16 \\
Unknown & 2 \\
Total & 55 \\
*here were 5 cases in unknown districts and 14 cases from districts outside Region 4. \\
\end{tabular}

Table 3. Updated district stillbirth rate

\begin{tabular}{lll}
\hline District & DHIS SBR & Adjusted SBR \\
\hline Umkhanyakude & 16.8 & 19.7 \\
Uthungulu & 24.3 & 19.9 \\
Zululand & 15.8 & 19.9 \\
SBR = stillbirth rate; DHIS = District Health Information System. &
\end{tabular}

Table 4 lists the outcomes and mode of delivery for 310 stillbirths during the review period.

Table 5 indicates the number of stillbirths distributed across weight categories for all 310 cases at LUWMRH compared with the average per regional hospital per year in the 9th Saving Babies report. ${ }^{[9]}$

Table 6 describes basic antenatal care demographics of the study population. 
Fig. 1 illustrates the number of patients with one or more adverse outcomes in previous pregnancies.

Fig. 2 shows the number of patients with the most common obstetric conditions associated with stillbirth. Some conditions occurred simultaneously in the same patient, e.g. $37 \%(n=40)$ of patients with abruptio placentae also had pre-existing gestational hypertension.

Fig. 3 demonstrates the number of patients who experienced the most common areas of substandard care at clinic level - some occurred simultaneously in the same patient.

Fig. 4 illustrates the number of patients who experienced the most common areas of substandard care at district and regional hospital level - some occurred simultaneously in the same patient.

With regard to the most common avoidable factors, $15 \%(n=45)$ of patients reported a late response to reduced fetal movements, and $9 \%$ $(n=27)$ experienced a delay in transfer to the referring facility, either from a clinic to the local district hospital or to LUWMRH.

\section{Discussion}

It is clear that the inflated averages for the SBR and SBR $\geq 1000 \mathrm{~g}$ at LUWMRH are due to referrals of high-risk and complicated cases from clinics and district hospitals in the region. Consequently, when calculating an average using the rates for the three districts that comprise Region 4, the SBR and SBR $\geq 1000 \mathrm{~g}$ are similar to national and provincial rates. Of the 310 patients, 291 came from facilities in Region 4 . When these cases are disaggregated per district, Uthungulu appears to have the largest proportion of stillbirths compared with the other two districts. However, 151 (52\%) of these patients were from surrounding primary healthcare clinics, which - in the absence of a level 1 district hospital - refer all their high-risk and emergency patients directly to LUWMRH. Had these patients been managed at a district hospital instead of at LUWMRH, the institutional SBR would possibly be lower. When the 103 patients from Umkhanyakude and Zululand districts, who delivered stillborn babies, were deducted from those from Uthungulu district and added to their referring district facility's stillbirth data from the DHIS, the rates for each district during the study period were almost equal. The adjusted in-facility stillbirth rates further illustrate the effect of referred cases on the institutional and district SBR for LUWMRH and Uthungulu district, respectively.

The referral system is also responsible for the high proportion of macerated stillborn infants, as well as the caesarean section rate

\begin{tabular}{ll}
\multicolumn{2}{l}{ Table 4. Stillbirth outcomes and mode of delivery, $\mathbf{N = 3 1 0}$} \\
\hline Stillbirth & $\boldsymbol{n}(\%)$ \\
\hline Outcome & \\
$\quad$ Macerated & $225(73)$ \\
Fresh & $85(27)$ \\
Mode of delivery & \\
$\quad$ Normal vaginal & $232(75)$ \\
Caesarean section & $78(25)$
\end{tabular}

of $25 \%$ in the case of stillbirths at LUWMRH, as this percentage includes intrauterine deaths (IUDs) in patients referred to the facility for induction of labour and for emergency cases that required immediate delivery. In addition, the number of stillbirths per weight category of $2000-2499 \mathrm{~g}$ is more than double the average per weight category per year at regional hospitals (Table 4) ${ }^{\left[{ }^{[9]}\right.}$ The total average number of stillbirths per year at regional hospitals was 173 of an average 5724 deliveries per year compared with 310 stillbirths of 8795 deliveries at LUWMRH in the 12-month review period. However, at LUWMRH there were 47 stillborn infants weighing $>2500 \mathrm{~g}$ - fewer than the average of 49 for regional hospitals.

Ideally, the SBR per category for maternal age, parity, antenatal care and number of visits to the antenatal clinic should be calculated to determine whether there are differences in the SBR within categories, but this was not possible, as the total number of deliveries per category is not routinely collected in the PPIP. Therefore, to enable comparison, it is recommended that follow-up audits should collect the same demographic information for all infants delivered.

Sixty-four $(21 \%)$ women in this sample had a previous adverse pregnancy outcome, which has been identified as a risk factor for stillbirth. ${ }^{[11,12]}$ Such patients need special attention and early antenatal care for future pregnancies. As a result, the obstetrics and gynaecology department at LUWMRH set up a specialist-run antenatal clinic for patients known to have had severe pre-eclampsia, unexplained IUDs and abruptio placentae.

The most common obstetric causes of stillbirth in this sample were similar to those in a study by Hossain et al. ${ }^{[13]}$ from a tertiary hospital in Pakistan, examining causes of stillbirth in low socioeconomic settings. Gestational hypertensive disorders

Table 6. Basic antenatal care demographics, $N=305$

\begin{tabular}{lll}
\hline Demographic variable & Category & $\boldsymbol{n}(\%)$ \\
\hline Maternal age, years & $<18$ & $14(5)$ \\
& $18-34$ & $257(84)$ \\
& $>34$ & $33(11)$ \\
Maternal parity, $n$ & 0 & 0 \\
& $1-4$ & $114(37)$ \\
Antenatal care & $>4$ & $185(61)$ \\
& Unknown & $6(2)$ \\
& Unbooked & 0 \\
Booked & $19(6)$ \\
Antenatal clinic visits, $n$ & 1 st visit $<20$ weeks & $286(94)$ \\
& Unknown & $2(1)$ \\
& None & $17(6)$ \\
& $1-3$ & $93(31)$ \\
& $\geq 4$ & $191(63)$ \\
& Unknown & 0
\end{tabular}

Table 5. Stillbirths per weight category, $n$

\begin{tabular}{|c|c|c|c|c|c|c|}
\hline Weight, $\mathrm{g}$ & $500-999$ & $1000-1499$ & $1500-1999$ & $2000-2499$ & $>2500$ & Total \\
\hline LUWMRH audit & 79 & 78 & 55 & 51 & 47 & 310 \\
\hline Regional hospitals $^{[9]}$ & 46 & 29 & 26 & 23 & 49 & 173 \\
\hline
\end{tabular}




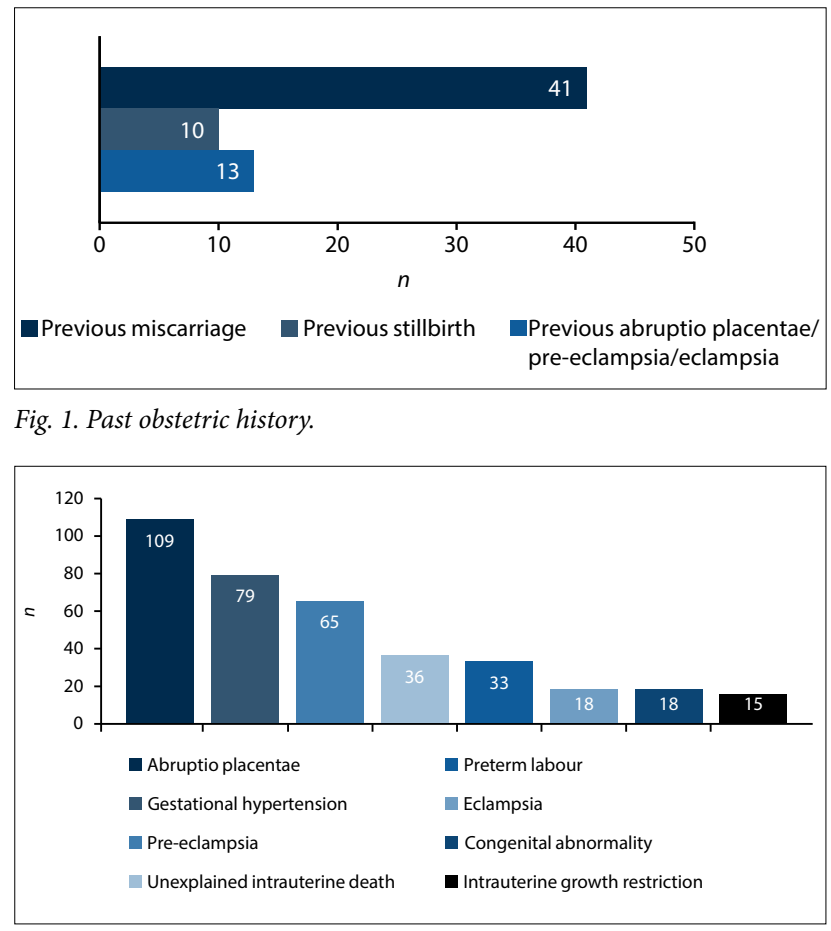

Fig. 2. Most common obstetric conditions associated with stillbirths.

and abruptio placentae with or without hypertension were major contributing factors to perinatal mortality in both samples. Unexplained IUDs (12\%) and congenital abnormalities (6\%) contributed to stillbirths in this sample, and were $11 \%$ and $7 \%$, respectively, in Hossain et al.'s ${ }^{[13]}$ sample. Although not shown in the results, only 5 cases were clearly identified as being the result of intrapartum asphyxia related to a delay in recognising fetal distress and a delay in immediate delivery. All 5 infants weighed $>2500 \mathrm{~g}$. Goldenberg et al. ${ }^{[14]}$ argued that intrapartum asphyxia as a cause of death is often determined by the clinical circumstances preceding death, and that underlying conditions such as eclampsia, abruptio placentae or intrauterine growth restriction are usually listed as the cause of death.

As elevated blood pressure and pre-eclampsia were identified as areas of substandard care at clinic level, and because of the significant proportion of gestational hypertensive diseases contributing to stillbirths, the management of these conditions have been flagged as a priority for skills development in the region. In November 2015, a regional workshop was held, aimed at improving healthcare workers' skills with regard to managing gestational hypertension. Since then, patients diagnosed with gestational hypertension have been closely monitored and a follow-up workshop is planned. The use of the growth chart in plotting symphysis-fundal height at each antenatal visit is important to identify intrauterine growth restriction, especially in patients with gestational hypertension; this must be routinely reinforced as part of basic antenatal care. ${ }^{[15]}$ At hospital level, the areas of substandard care identified are an indication of the need for ongoing training in the Essential Steps in the Management of Obstetric Emergencies (ESMOE), and regular outreach and feedback to surrounding district hospitals regarding patients referred to LUWMRH.

Besides enquiring about fetal movements during basic antenatal clinic visits, the Guidelines for Maternity Care in South Africa ${ }^{[15]}$ recommend that active fetal movement monitoring with the use of a fetal movement chart should be commenced when the fetus is viable after 28 weeks in cases of gestational hypertension, mild-to-moderate

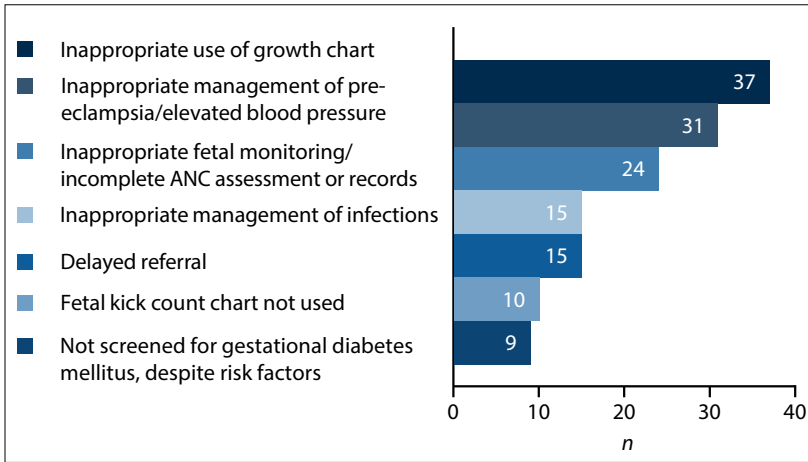

Fig. 3. Areas of substandard care at clinic level. $(A N C=$ antenatal care. $)$

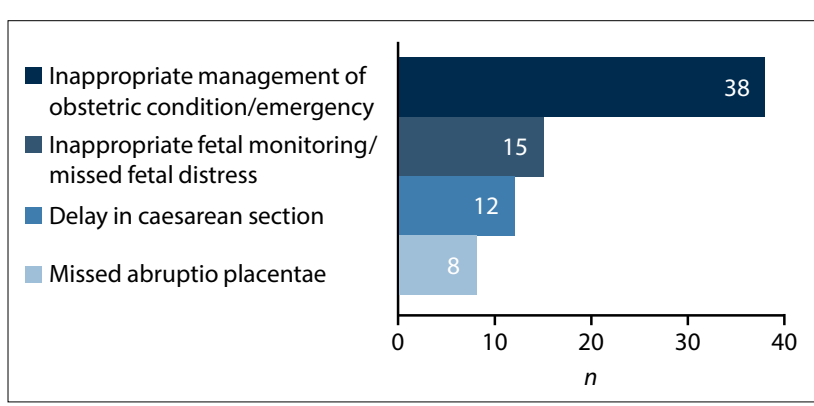

Fig. 4. Areas of substandard care at hospital level.

pre-eclampsia, intrauterine growth restriction and antepartum haemorrhage of unknown origin (after excluding abruptio placentae and placenta praevia). There is a gap in the literature regarding the implementation and acceptance of this chart and its effectiveness in reducing stillbirths in SA. A systematic review of interventions to improve maternal awareness of decreased fetal movement, based on 16 studies from developed countries, did not provide clear evidence of benefit or harm; however, indirect evidence suggested improved pregnancy and birth outcomes. ${ }^{[16]}$ Olabuji et al.${ }^{[17]}$ demonstrated that maternal education level was a significant factor in the perception of abnormal fetal movements in their sample of Nigerian women during the third trimester of pregnancy. However, $41 \%$ of the women indicated they would only seek help after 24 hours of absent fetal movements. The authors concluded that this delay may be associated with poor knowledge of extreme complications, such as IUD associated with reduced fetal activity, as only $16.4 \%$ of their sample were aware of IUD as a complication. The association between low levels of education or socioeconomic status and poor antenatal attendance in developing countries has been reported previously. ${ }^{[18-20]}$ Ha et al..$^{[8]}$ found that reduced antenatal attendance increased the risk of stillbirth, as poorer women receive substandard care and are unable to use services effectively owing to a lack of resources. Such services are necessary to follow recommendations for health-seeking behaviour. Considering the socioeconomic status of the population in Region 4 of KZN, further research is needed to better describe the knowledge of fetal movements among pregnant women and develop appropriately targeted messages for this population.

Since the completion of the audit, a number of interventions have been implemented. Emergency medical response services (EMRSs) have been strengthened to address the delay in transportation between facilities in the region. EMRS management personnel are invited to attend the weekly district perinatal morbidity and mortality meetings to receive and provide feedback on cases involving a delay in transport. It is important to note that such a delay often underscores the environmental problems of rural SA, such as long distances between facilities, dirt roads and poor visibility at night, 
coupled with resource challenges, such as a shortage of vehicles and trained paramedics. Where possible, during the day the SA Red Cross Air Mercy Service is employed.

\section{Study limitations}

Although the results presented were obtained from the HUMMET tool, a major limitation to this study was missing or incomplete forms, which has most likely resulted in an underestimation of the findings and an omission of details regarding the circumstances around certain conditions such as abruptio placentae and descriptions of congenital abnormalities. After the initial presentation of these findings, a more reliable system has been implemented to ensure that each form is completed and captured electronically on a daily basis, with a copy being sent to the medical manager of the hospital. More research is needed to further investigate the cases of unexplained IUDs. Dedicated resources are required to conduct histopathology studies and autopsies on these cases.

\section{Conclusion}

The HUMMET form provides a systematic approach to analysing cases of perinatal morbidity and mortality in line with the requirements of the PPIP database, with more details on the circumstances and contributing factors. The audit of HUMMET forms has provided valuable information on the severity and volume of complicated obstetric cases referred to LUWMRH. However, it is recommended that a repeat audit be conducted to determine the effectiveness of subsequent interventions. Further quantitative and qualitative research is needed to better understand the obstacles that women encounter in this context - from early booking to health-seeking behaviour.

Acknowledgements. The author wishes to acknowledge Ms Sthandwa Mnqayi for her assistance in obtaining provincial perinatal statistics, as well as the Department of Obstetrics and Gynaecology, LUWMRH for support with the audit.

Author contributions. Sole author.

Funding. Self funded.

Conflicts of interest. None.
1. World Health Organization. Stillbirths. 2015. http://www.who.int/maternal_child_adolescent/epidemiology/ stillbirth/en/ (accessed 25 October 2017).

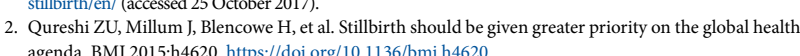

3. Aminu M, Unkels R, Mdegela M, Utz B, Adaji S, van den Broek N. Causes of and factors associated Aminu M, Unkels R, Mdegela M, Utz B, Adaji S, van den Broek N. Causes of and factors associated
with stillbirth in low- and middle-income countries: A systematic literature review. Br J Obstet Gynaecol 2014;121(Suppl 4):141-153. https://doi.org/10.1111/1471-0528.12995

4. Lawn J, Blencowe H, Pattinson R, et al. Stillbirths: Where? When? Why? How to make the data count. Lancet 2011;377(9775):1448-1463. https://doi.org/10.1016/S0140-6736(10)62187-3

5. Goldenberg R, McClure E, Bhutta Z, et al. Stillbirths: The vision for 2020. Lancet 2011;377(9779):1798 1805. https://doi.org/10.1016/S0140-6736(10)62235-0

6. McClure E, Saleem S, Pasha O, Goldenberg R. Stillbirth in developing countries: A review of causes, risk factors and prevention strategies. J Matern Fetal Neonatal Med 2009;22(3):183-190. https://doi. org/10.1080/14767050802559129

7. McClure E, Pasha O, Goudar S, et al. Epidemiology of stillbirth in low-middle income countries: A global network study. Acta Obstet Gynecol Scand 2011;90(12):1379-1385. https://doi.org/10.1111/ j.1600-0412.2011.01275.x

8. Ha Y, Hurt L, Tawiah-Agyemang C, Kirkwood B, Edmond K. Effect of socioeconomic deprivation and health service utilisation on antepartum and intrapartum stillbirth: Population cohort study from rural Ghana. PLoS ONE 2012;7(7):e39050. https://doi.org/10.1371/journal.pone.0039050

9. Pattinson RC, Rhoda N. Saving Babies 2012 - 2013: Ninth Report on Perinatal Care in South Africa. Pretoria: Tshepesa Press, 2014.

10. Massyn N, Peer N, Padarath A, Barron P, Day C. District Health Barometer 2014/15. Durban: Health Systems Trust, 2015.

11. Oladapo OT, Adekanle DA, Durojaiye BO. Maternal risk factors associated with fetal death during antenatal care in low-resource tertiary hospitals. Aust N Z J Obstet Gynaecol 2007;47(5):383-388. https://doi.org/10.1111/j.1479-828X.2007.00761.x

12. Measey MA, d'Espaignet ET, Charles A, Douglass C. Unexplained fetal death: Are women with a history of fetal loss at higher risk? Aust N Z J Obstet Gynaecol 2009;49(2):151-157. https://doi org/10.1111/j.1479-828X.2009.00982.x

13. Hossain N, Khan N, Khan NH. Obstetric causes of stillbirth at low socioeconomic settings. J Pakistan Med Assoc 2009;59(11):744-747.

14. Goldenberg RL, Harrison MS, McClure EM. Stillbirths: The hidden birth asphyxia - US and global perspectives. Clin Perinatol 2016;43(3):439-453. https://doi.org/10.1016/j.clp.2016.04.004

15. National Department of Health. Guidelines for Maternity Care in South Africa: A Manual for Clinics, Community Health Centres and District Hospitals. Pretoria: NDoH, 2015.
.

16. Winje BA, Wojcieszek AM, Gonzalez-Angulo LY, et al. Interventions to enhance maternal awareness of decreased fetal movement: A systematic review. Br J Obstet Gynaecol 2016;123(6):886-898. https:// doi.org/10.1111/1471-0528.13802

17. Olagbuji B, Igbarumah S, Akintayo A, Olofinbiyi B, Aduloju P, Alao O. Maternal understanding of fetal movement in third trimester: A means for fetal monitoring and reducing stillbirth. Niger J Clin Pract 2014;17(4):489. https://doi.org/10.4103/1119-3077.134049

18. Arthur E. Wealth and antenatal care use: Implications for maternal health care utilisation in Ghana. Health Econ Rev 2012;2(1):14. https://doi.org/10.1186/2191-1991-2-14

19. Fagbamigbe AF, Idemudia ES. Barriers to antenatal care use in Nigeria: Evidences from non-users and implications for maternal health programming. BMC Preg Childbirth 2015;15(1):95. https://doi. org/10.1186/s12884-015-0527-y

20. Joshi C, Torvaldsen S, Hodgson R, Hayen A. Factors associated with the use and quality of antenatal care in Nepal: A population-based study using the demographic and health survey data. BMC Preg Childbirth 2014;14(1):94. https://doi.org/10.1186/1471-2393-14-94 


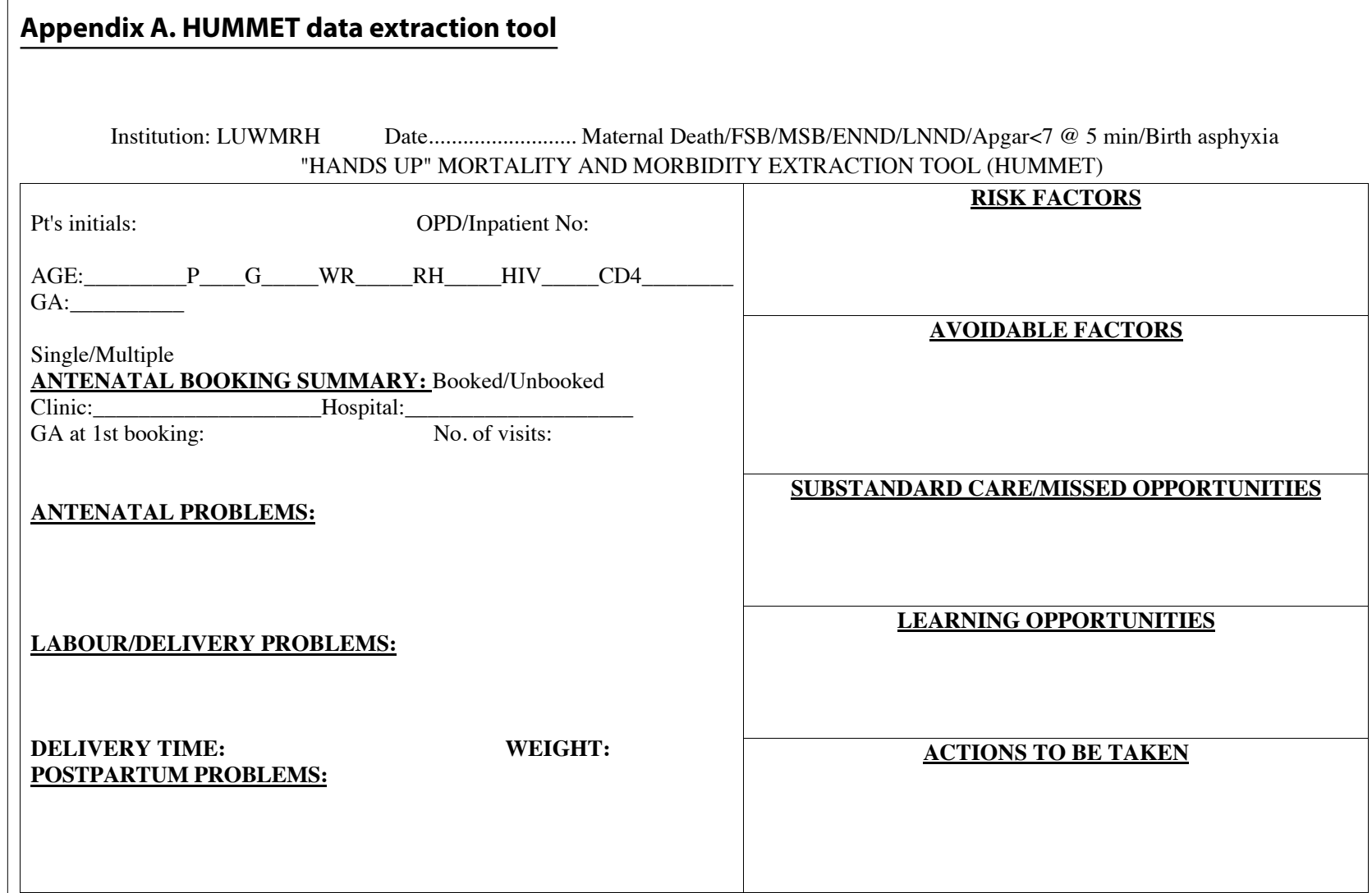

Consultant ward round done by: $\quad$ BI form filled by: 